\title{
Allogeneic Bone Marrow Transplantation Recipient
}

National Cancer Institute

\section{Source}

National Cancer Institute. Allogeneic Bone Marrow Transplantation Recipient. NCI

Thesaurus. Code C161848.

An individual receiving an allogeneic bone marrow transplant. 